\title{
Combinatorial Properties and Characterization of Glued Semigroups
}

\author{
J. I. García-García, ${ }^{1}$ M. A. Moreno-Frías, ${ }^{1}$ and A. Vigneron-Tenorio ${ }^{2}$ \\ ${ }^{1}$ Departamento de Matemáticas, Universidad de Cádiz, Puerto Real, 11510 Cádiz, Spain \\ ${ }^{2}$ Departamento de Matemáticas, Universidad de Cádiz, Jerez de la Frontera, 11405 Cádiz, Spain
}

Correspondence should be addressed to M. A. Moreno-Frías; mariangeles.mfrias@gmail.com

Received 26 September 2013; Accepted 12 October 2013; Published 13 March 2014

Academic Editor: Maria Gandarias

Copyright ( 2014 J. I. García-García et al. This is an open access article distributed under the Creative Commons Attribution License, which permits unrestricted use, distribution, and reproduction in any medium, provided the original work is properly cited.

This work focuses on the combinatorial properties of glued semigroups and provides its combinatorial characterization. Some classical results for affine glued semigroups are generalized and some methods to obtain glued semigroups are developed.

\section{Introduction}

Let $S=\left\langle n_{1}, \ldots, n_{l}\right\rangle$ be a finitely generated commutative semigroup with zero element which is reduced (i.e., $S \cap(-S)=\{0\}$ ) and cancellative (if $m, n, n^{\prime} \in S$ and $m+n=m+n^{\prime}$ then $\left.n=n^{\prime}\right)$. Under these settings if $S$ is torsion-free, then it is isomorphic to a subsemigroup of $\mathbb{N}^{p}$ which means it is an affine semigroup (see [1]). From now on assume that all the semigroups appearing in this work are finitely generated, commutative, reduced, and cancellative, but not necessarily torsionfree.

Let $\mathbb{K}$ be a field and $\mathbb{K}\left[X_{1}, \ldots, X_{l}\right]$ the polynomial ring in $l$ indeterminates. This polynomial ring is obviously an $S$ graded ring (by assigning the $S$-degree $n_{i}$ to the indeterminate $X_{i}$, the $S$-degree of $X^{\alpha}=X_{1}^{\alpha_{1}} \cdots X_{l}^{\alpha_{l}}$ is $\left.\sum_{i=1}^{l} \alpha_{i} n_{i} \in S\right)$. It is well known that the ideal $I_{S}$ generated by

$$
\left\{X^{\alpha}-X^{\beta} \mid \sum_{i=1}^{l} \alpha_{i} n_{i}=\sum_{i=1}^{l} \beta_{i} n_{i}\right\} \subset \mathbb{K}\left[X_{1}, \ldots, X_{l}\right]
$$

is an $S$-homogeneous binomial ideal called semigroup ideal (see [2] for details). If $S$ is torsion-free, the ideal obtained defines a toric variety (see [3] and the references therein). By Nakayama's lemma, all the minimal generating sets of $I_{S}$ have the same cardinality and the $S$-degrees of its elements can be determinated.

The main goal of this work is to study the semigroups which result from the gluing of other two. This concept was introduced by Rosales in [4] and it is closely related to complete intersection ideals (see [5] and the references therein). A semigroup $S$ minimally generated by $A_{1} \sqcup A_{2}$ (with $A_{1}=$ $\left\{n_{1}, \ldots, n_{r}\right\}$ and $\left.A_{2}=\left\{n_{r+1}, \ldots, n_{l}\right\}\right)$ is the gluing of $S_{1}=\left\langle A_{1}\right\rangle$ and $S_{2}=\left\langle A_{2}\right\rangle$ if there exists a set of generators $\rho$ of $I_{S}$ of the form $\rho=\rho_{1} \cup \rho_{2} \cup\left\{X^{\gamma}-X^{\gamma^{\prime}}\right\}$, where $\rho_{1}, \rho_{2}$ are generating sets of $I_{S_{1}}$ and $I_{S_{2}}$, respectively, $X^{\gamma}-X^{\gamma^{\prime}} \in I_{S}$, and the supports of $\gamma$ and $\gamma^{\prime}$ verify $\operatorname{supp}(\gamma) \subset\{1, \ldots, r\}$ and $\operatorname{supp}\left(\gamma^{\prime}\right) \subset\{r+1, \ldots, l\}$. Equivalently, $S$ is the gluing of $S_{1}$ and $S_{2}$ if $I_{S}=I_{S_{1}}+I_{S_{2}}+\left\langle X^{\gamma}-\right.$ $\left.X^{\gamma^{\prime}}\right\rangle$. A semigroup is a glued semigroup when it is the gluing of other two.

As seen, glued semigroups can be determinated by the minimal generating sets of $I_{S}$ which can be studied by using combinatorial methods from certain simplicial complexes (see [6-8]). In this work the simplicial complexes used are defined as follows: for any $m \in S$, set

$$
C_{m}=\left\{X^{\alpha}=X_{1}^{\alpha_{1}} \cdots X_{l}^{\alpha_{l}} \mid \sum_{i=1}^{l} \alpha_{i} n_{i}=m\right\},
$$

and the simplicial complex

$$
\nabla_{m}=\left\{F \subseteq C_{m} \mid \operatorname{gcd}(F) \neq 1\right\},
$$

with $\operatorname{gcd}(F)$ as the greatest common divisor of the monomials in $F$. 
Furthermore, some methods which require linear algebra and integer programming are given to obtain examples of glued semigroups.

The content of this work is organized as follows. Section 2 presents the tools to generalize to nontorsion-free semigroups a classical characterization of affine gluing semigroups (Proposition 2). In Section 3, the nonconnected simplicial complexes $\nabla_{m}$ associated with glued semigroups are studied. By using the vertices of the connected components of these complexes we give a combinatorial characterization of glued semigroups as well as their glued degrees (Theorem 6). Besides, in Corollary 7 we deduce the conditions for the ideal of a glued semigroup to have a unique minimal system of generators. Finally, Section 4 is devoted to the construction of glued semigroups (Corollary 10) and affine glued semigroups (Section 4.1).

\section{Preliminaries and Generalizations about Glued Semigroups}

A binomial of $I_{S}$ is called indispensable if it is an element of all systems of generators of $I_{S}$ (up to a scalar multiple). This kind of binomials was introduced in [9] and they have an important role in Algebraic Statistics. In [10] the authors characterize indispensable binomials by using simplicial complexes $\nabla_{m}$. Note that if $I_{S}$ is generated by its indispensable binomials then it is minimally generated, up to scalar multiples, in an unique way.

With the above notation, the semigroup $S$ is associated with the lattice $\operatorname{ker} S$ formed by the elements $\alpha=$ $\left(\alpha_{1}, \ldots, \alpha_{l}\right) \in \mathbb{Z}^{l}$ such that $\sum_{i=1}^{l} \alpha_{i} n_{i}=0$. Given $G$ a system of generators of $I_{S}$, the lattice ker $S$ is generated by the elements $\alpha-\beta$ with $X^{\alpha}-X^{\beta} \in G$ and ker $S$ also verifies that $\operatorname{ker} S \cap \mathbb{N}^{l}=$ $\{0\}$ if and only if $S$ is reduced. If $\mathscr{M}\left(I_{S}\right)$ is a minimal generating set of $I_{S}$, denote by $\mathscr{M}\left(I_{S}\right)_{m} \subset \mathscr{M}\left(I_{S}\right)$ the set of elements whose $S$-degree is equal to $m \in S$ and by $\operatorname{Betti}(S)$ the set of the $S$-degrees of the elements of $\mathscr{M}\left(I_{S}\right)$. When $I_{S}$ is minimally generated by $\operatorname{rank}(\operatorname{ker} S)$ elements, the semigroup $S$ is called a complete intersection semigroup.

Let $\mathscr{C}\left(\nabla_{m}\right)$ be the number of connected components of $\nabla_{m}$. The cardinality of $\mathscr{M}\left(I_{S}\right)_{m}$ is equal to $\mathscr{C}\left(\nabla_{m}\right)-1$ (see Remark 2.6 in [6] and Theorem 3 and Corollary 4 in [8]) and the complexes associated with the elements in $\operatorname{Betti}(S)$ are nonconnected.

Construction 1 (see [7, Proposition 1]). For each $m \in \operatorname{Betti}(S)$ the set $\mathscr{M}\left(I_{S}\right)_{m}$ is obtained by taking $\mathscr{C}\left(\nabla_{m}\right)-1$ binomials with monomials in different connected components of $\nabla_{m}$ satisfying that two different binomials do not have their corresponding monomials in the same components and fulfilling that there is at least a monomial of every connected component of $\nabla_{m}$. This let us construct a minimal generating set of $I_{S}$ in a combinatorial way.

Let $S$ be minimally (we consider a minimal generator set of $S$ and in the other case $S$ is trivially the gluing of the semigroup generated by one of its nonminimal generators and the semigroup generated by the others) generated by
$A_{1} \sqcup A_{2}$ with $A_{1}=\left\{a_{1}, \ldots, a_{r}\right\}$ and $A_{2}=\left\{b_{1}, \ldots, b_{t}\right\}$. From now on, identify the sets $A_{1}$ and $A_{2}$ with the matrices $\left(a_{1}|\cdots| a_{r}\right)$ and $\left(b_{1}|\cdots| b_{t}\right)$. Denote by $\mathbb{K}\left[A_{1}\right]$ and $\mathbb{K}\left[A_{2}\right]$ the polynomial rings $\mathbb{K}\left[X_{1}, \ldots, X_{r}\right]$ and $\mathbb{K}\left[Y_{1}, \ldots, Y_{t}\right]$, respectively. A monomial is a pure monomial if it has indeterminates only in $X_{1}, \ldots, X_{r}$ or only in $Y_{1}, \ldots, Y_{t}$; otherwise it is a mixed monomial. If $S$ is the gluing of $S_{1}=\left\langle A_{1}\right\rangle$ and $S_{2}=\left\langle A_{2}\right\rangle$, then the binomial $X^{\gamma_{X}}-Y^{\gamma_{Y}} \in I_{S}$ is a glued binomial if $\mathscr{M}\left(I_{S_{1}}\right) \cup \mathscr{M}\left(I_{S_{2}}\right) \cup\left\{X^{\gamma_{X}}-Y^{\gamma_{Y}}\right\}$ is a generating set of $I_{S}$ and in this case the element $d=S$-degree $\left(X^{\gamma_{X}}\right) \in S$ is called a glued degree.

It is clear that if $S$ is a glued semigroup, the lattice $\operatorname{ker} S$ has a basis of the form

$$
\left\{L_{1}, L_{2},\left(\gamma_{X},-\gamma_{Y}\right)\right\} \subset \mathbb{Z}^{r+t},
$$

where the supports of the elements in $L_{1}$ are in $\{1, \ldots, r\}$, the supports of the elements in $L_{2}$ are in $\{r+1, \ldots, r+t\}$, $\operatorname{ker} S_{i}=$ $\left\langle L_{i}\right\rangle(i=1,2)$ by considering only the coordinates in $\{1, \ldots$, $r\}$ or $\{r+1, \ldots, r+t\}$ of $L_{i}$, and $\left(\gamma_{X}, \gamma_{Y}\right) \in \mathbb{N}^{r+t}$. Moreover, since $S$ is reduced, one has that $\left\langle L_{1}\right\rangle \cap \mathbb{N}^{r+t}=\left\langle L_{2}\right\rangle \cap \mathbb{N}^{r+t}=\{0\}$. Denote by $\left\{\rho_{1 i}\right\}_{i}$ the elements in $L_{1}$ and by $\left\{\rho_{2 i}\right\}_{i}$ the elements in $L_{2}$.

The following proposition generalizes [4, Theorem 1.4] to nontorsion-free semigroups.

Proposition 2. The semigroup $S$ is the gluing of $S_{1}$ and $S_{2}$ if and only if there exists $d \in\left(S_{1} \cap S_{2}\right) \backslash\{0\}$ such that $G\left(S_{1}\right) \cap$ $G\left(S_{2}\right)=d \mathbb{Z}$, where $G\left(S_{1}\right), G\left(S_{2}\right)$, and $d \mathbb{Z}$ are the associated commutative groups of $S_{1}, S_{2}$, and $\{d\}$, respectively.

Proof. Assume that $S$ is the gluing of $S_{1}$ and $S_{2}$. In this case, ker $S$ is generated by the set (4). Since $\left(\gamma_{X},-\gamma_{Y}\right) \in \operatorname{ker} S$, the element $d$ is equal to $A_{1} \gamma_{X}=A_{2} \gamma_{Y} \in S$ and $d \in S_{1} \cap S_{2} \subset$ $G\left(S_{1}\right) \cap G\left(S_{2}\right)$. Let $d^{\prime}$ be in $G\left(S_{1}\right) \cap G\left(S_{2}\right)$; then there exists $\left(\delta_{1}, \delta_{2}\right) \in \mathbb{Z}^{r} \times \mathbb{Z}^{t}$ such that $d^{\prime}=A_{1} \delta_{1}=A_{2} \delta_{2}$. Therefore $\left(\delta_{1},-\delta_{2}\right) \in \operatorname{ker} S$ because $\left(A_{1} \mid A_{2}\right)\left(\delta_{1},-\delta_{2}\right)=0$ and so there exist $\lambda, \lambda_{i}^{\rho_{1}}, \lambda_{i}^{\rho_{2}} \in \mathbb{Z}$ satisfying

$$
\begin{gathered}
\left(\delta_{1}, 0\right)=\sum_{i} \lambda_{i}^{\rho_{1}} \rho_{1 i}+\lambda\left(\gamma_{X}, 0\right), \\
\left(0, \delta_{2}\right)=-\sum_{i} \lambda_{i}^{\rho_{2}} \rho_{2 i}+\lambda\left(0, \gamma_{Y}\right),
\end{gathered}
$$

and $d^{\prime}=A_{1} \delta_{1}=\sum_{i} \lambda_{i}^{\rho_{1}}\left(A_{1} \mid 0\right) \rho_{1 i}+\lambda A_{1} \gamma_{X}=\lambda d$. We conclude that $G\left(S_{1}\right) \cap G\left(S_{2}\right)=d \mathbb{Z}$ with $d \in S_{1} \cap S_{2}$.

Conversely, suppose that there exists $d \in\left(S_{1} \cap S_{2}\right) \backslash\{0\}$ such that $G\left(S_{1}\right) \cap G\left(S_{2}\right)=d \mathbb{Z}$. We see that $I_{S}=I_{S_{1}}+I_{S_{2}}+\left\langle X^{\gamma_{X}}\right.$ $\left.Y^{\gamma_{Y}}\right\rangle$. Trivially, $I_{S_{1}}+I_{S_{2}}+\left\langle X^{\gamma_{X}}-Y^{\gamma_{Y}}\right\rangle \subset I_{S}$. Let $X^{\alpha} Y^{\beta}-X^{\gamma} Y^{\delta}$ be a binomial in $I_{S}$. Its $S$-degree is $A_{1} \alpha+A_{2} \beta=A_{1} \gamma+A_{2} \delta$. Using $A_{1}(\alpha-\gamma)=A_{2}(\beta-\delta) \in G\left(S_{1}\right) \cap G\left(S_{2}\right)=d \mathbb{Z}$, there exists $\lambda \in \mathbb{Z}$ such that $A_{1} \alpha=A_{1} \gamma+\lambda d$ and $A_{2} \delta=A_{2} \beta+\lambda d$. We have the following cases.

(i) If $\lambda=0$,

$$
\begin{aligned}
X^{\alpha} Y^{\beta}-X^{\gamma} Y^{\delta} & =X^{\alpha} Y^{\beta}-X^{\gamma} Y^{\beta}+X^{\gamma} Y^{\beta}-X^{\gamma} Y^{\delta} \\
& =Y^{\beta}\left(X^{\alpha}-X^{\gamma}\right)+X^{\gamma}\left(Y^{\beta}-Y^{\delta}\right) \in I_{S_{1}}+I_{S_{2}} .
\end{aligned}
$$


(ii) If $\lambda>0$,

$$
\begin{aligned}
X^{\alpha} Y^{\beta}-X^{\gamma} Y^{\delta}= & X^{\alpha} Y^{\beta}-X^{\gamma} X^{\lambda \gamma_{X}} Y^{\beta} \\
& +X^{\gamma} X^{\lambda \gamma_{X}} Y^{\beta}-X^{\gamma} X^{\lambda \gamma_{Y}} Y^{\beta}+X^{\gamma} X^{\lambda \gamma_{Y}} Y^{\beta} \\
& -X^{\gamma} Y^{\delta}=Y^{\beta}\left(X^{\alpha}-X^{\gamma} X^{\lambda \gamma_{X}}\right) \\
& +X^{\gamma} Y^{\beta}\left(X^{\lambda \gamma_{X}}-Y^{\lambda \gamma_{Y}}\right) \\
& +X^{\gamma}\left(Y^{\lambda \gamma_{Y}} Y^{\beta}-Y^{\delta}\right) .
\end{aligned}
$$

Using that

$$
X^{\lambda \gamma_{X}}-Y^{\lambda \gamma_{Y}}=\left(X^{\gamma_{X}}-Y^{\gamma_{Y}}\right)\left(\sum_{i=0}^{\lambda-1} X^{(\lambda-1-i) \gamma_{X}} Y^{i \gamma_{Y}}\right)
$$

the binomial $X^{\alpha} Y^{\beta}-X^{\gamma} Y^{\delta}$ belongs to $I_{S_{1}}+I_{S_{2}}+\left\langle X^{\gamma_{X}}-\right.$ $\left.Y^{\gamma_{Y}}\right\rangle$.

(iii) The case $\lambda<0$ is solved similarly.

We conclude that $I_{S}=I_{S_{1}}+I_{S_{2}}+\left\langle X^{\gamma_{X}}-Y^{\gamma_{Y}}\right\rangle$.

From the above proof it is deduced that given the partition of the system of generators of $S$ the glued degree is unique.

\section{Glued Semigroups and Combinatorics}

Glued semigroups by means of nonconnected simplicial complexes are characterized. For any $m \in S$, redefine $C_{m}$ from (2) as

$C_{m}=\left\{X^{\alpha} Y^{\beta}=X_{1}^{\alpha_{1}} \cdots X_{r}^{\alpha_{r}} Y_{1}^{\beta_{1}} \cdots Y_{t}^{\beta_{t}} \mid \sum_{i=1}^{r} \alpha_{i} a_{i}+\sum_{i=1}^{t} \beta_{i} b_{i}=m\right\}$,

and consider the sets of vertices and the simplicial complexes

$$
\begin{gathered}
C_{m}^{A_{1}}=\left\{X_{1}^{\alpha_{1}} \cdots X_{r}^{\alpha_{r}} \mid \sum_{i=1}^{r} \alpha_{i} a_{i}=m\right\}, \\
\nabla_{m}^{A_{1}}=\left\{F \subseteq C_{m}^{A_{1}} \mid \operatorname{gcd}(F) \neq 1\right\}, \\
C_{m}^{A_{2}}=\left\{Y_{1}^{\beta_{1}} \cdots Y_{t}^{\beta_{t}} \mid \sum_{i=1}^{t} \beta_{i} b_{i}=m\right\}, \\
\nabla_{m}^{A_{2}}=\left\{F \subseteq C_{m}^{A_{2}} \mid \operatorname{gcd}(F) \neq 1\right\},
\end{gathered}
$$

where $A_{1}=\left\{a_{1}, \ldots, a_{r}\right\}$ and $A_{2}=\left\{b_{1}, \ldots, b_{t}\right\}$ as in Section 2 . Trivially, the relations between $\nabla_{m}^{A_{1}}, \nabla_{m}^{A_{2}}$, and $\nabla_{m}$ are

$$
\nabla_{m}^{A_{1}}=\left\{F \in \nabla_{m} \mid F \subset C_{m}^{A_{1}}\right\}, \quad \nabla_{m}^{A_{2}}=\left\{F \in \nabla_{m} \mid F \subset C_{m}^{A_{2}}\right\} .
$$

The following result shows an important property of the simplicial complexes associated with glued semigroups.
Lemma 3. Let $S$ be the gluing of $S_{1}$ and $S_{2}$ and $m \in \operatorname{Betti}(S)$. Then all the connected components of $\nabla_{m}$ have at least a pure monomial. In addition, all mixed monomials of $\nabla_{m}$ are in the same connected component.

Proof. Suppose that there exists $C$, a connected component of $\nabla_{m}$ only with mixed monomials. By Construction 1 in all generating sets of $I_{S}$ there is at least a binomial with a mixed monomial, but this does not occur in $\mathscr{M}\left(I_{S_{1}}\right) \cup \mathscr{M}\left(I_{S_{2}}\right) \cup\left\{X^{\gamma_{X}}-\right.$ $\left.Y^{\gamma_{Y}}\right\}$ with $X^{\gamma_{X}}-Y^{\gamma_{Y}}$ as a glued binomial.

Since $S$ is a glued semigroup, $\operatorname{ker} S$ has a system of generators as (4). Let $X^{\alpha} Y^{\beta}, X^{\gamma} Y^{\delta} \in C_{m}$ be two monomials such that $\operatorname{gcd}\left(X^{\alpha} Y^{\beta}, X^{\gamma} Y^{\delta}\right)=1$. In this case, $(\alpha, \beta)-(\gamma, \delta) \epsilon$ ker $S$ and there exist $\lambda, \lambda_{i}^{\rho_{1}}, \lambda_{i}^{\rho_{2}} \in \mathbb{Z}$ satisfying

$$
\begin{aligned}
& (\alpha-\gamma, 0)=\sum_{i} \lambda_{i}^{\rho_{1}} \rho_{1 i}+\lambda\left(\gamma_{X}, 0\right), \\
& (0, \beta-\delta)=\sum_{i} \lambda_{i}^{\rho_{2}} \rho_{2 i}-\lambda\left(0, \gamma_{Y}\right) .
\end{aligned}
$$

(i) If $\lambda=0, \alpha-\gamma \in \operatorname{ker} S_{1}$, and $\beta-\delta \in \operatorname{ker} S_{2}$, then $A_{1} \alpha=A_{1} \gamma, A_{2} \beta=A_{2} \delta$, and $X^{\alpha} Y^{\delta} \in C_{m}$.

(ii) If $\lambda>0,(\alpha, 0)=\sum_{i} \lambda_{i}^{\rho_{1}} \rho_{1 i}+\lambda\left(\gamma_{X}, 0\right)+(\gamma, 0)$, and

$$
A_{1} \alpha=\sum_{i} \lambda_{i}^{\rho_{1}}\left(A_{1} \mid 0\right) \rho_{1 i}+\lambda A_{1} \gamma_{X}+A_{1} \gamma=\lambda d+A_{1} \gamma
$$

$$
\text { then } X^{\lambda \gamma_{X}} X^{\gamma} Y^{\beta} \in C_{m} \text {. }
$$

(iii) The case $\lambda<0$ is solved likewise.

In any case, $X^{\alpha} Y^{\beta}$ and $X^{\gamma} Y^{\delta}$ are in the same connected component of $\nabla_{m}$.

We now describe the simplicial complexes that correspond to the $S$-degrees which are multiples of the glued degree.

Lemma 4. Let $S$ be the gluing of $S_{1}$ and $S_{2}, d \in S$ the glued degree, and $d^{\prime} \in S \backslash\{d\}$. Then $C_{d^{\prime}}^{A_{1}} \neq \emptyset \neq C_{d^{\prime}}^{A_{2}}$ if and only if $d^{\prime} \in(d \mathbb{N}) \backslash\{0\}$. Furthermore, the simplicial complex $\nabla_{d^{\prime}}$ has at least one connected component with elements in $C_{d^{\prime}}^{A_{1}}$ and $C_{d^{\prime}}^{A_{2}}$.

Proof. If there exist $X^{\alpha}, Y^{\beta} \in C_{d^{\prime}}$, then $d^{\prime}=\sum_{i=1}^{r} \alpha_{i} a_{i}=$ $\sum_{i=1}^{t} \beta_{i} b_{i} \in S_{1} \cap S_{2} \subset G\left(S_{1}\right) \cap G\left(S_{2}\right)=d \mathbb{Z}$. Hence, $d^{\prime} \in d \mathbb{N}$.

Conversely, let $d^{\prime}=j d$ with $j \in \mathbb{N}$ and $j>1$ and let $X^{\gamma_{X}}-Y^{\gamma_{Y}} \in I_{S}$ be a glued binomial. It is easy to see that $X^{j \gamma_{X}}, Y^{j \gamma_{Y}} \in C_{d^{\prime}}$ and thus $\left\{X^{j \gamma_{X}}, X^{(j-1) \gamma_{X}} Y^{\gamma_{Y}}\right\}$ and $\left\{X^{(j-1) \gamma_{X}} Y^{\gamma_{Y}}, Y^{j \gamma_{Y}}\right\}$ belong to $\nabla_{d^{\prime}}$.

The following lemma is a combinatorial version of [11, Lemma 9] and it is a necessary condition of Theorem 6.

Lemma 5. Let $S$ be the gluing of $S_{1}$ and $S_{2}$ and $d \in S$ the glued degree. Then the elements of $C_{d}$ are pure monomials and $d \epsilon$ $\operatorname{Betti}(S)$. 
Proof. The order $\unlhd_{S}$ defined by $m^{\prime} \unlhd_{S} m$ if $m-m^{\prime} \in S$ is a partial order on $S$.

Assume that there exists a mixed monomial $T \in C_{d}$. By Lemma 3, there exists a pure monomial $Y^{b}$ in $C_{d}$ such that $\left\{T, Y^{b}\right\} \in \nabla_{d}$ (the proof is analogous if we consider $X^{a}$ with $\left\{T, X^{a}\right\} \in \nabla_{d}$. Now take $T_{1}=\operatorname{gcd}\left(T, Y^{b}\right)^{-1} T$ and $Y^{b_{1}}=$ $\operatorname{gcd}\left(T, Y^{b}\right)^{-1} Y^{b}$. Both monomials are in $C_{d^{\prime}}$, where $d^{\prime}$ is equal to $d$ minus the $S$-degree of $\operatorname{gcd}\left(T, Y^{b}\right)$. By Lemma 4 , if $C_{d^{\prime}}^{A_{1}} \neq \emptyset$, then $d^{\prime} \in d \mathbb{N}$, but since $d^{\prime} \prec_{S} d$ this is not possible. So, if $T_{1}$ is a mixed monomial and $C_{d^{\prime}}^{A_{1}}=\emptyset$, then $C_{d^{\prime}}^{A_{2}} \neq \emptyset$. If there exists a pure monomial in $C_{d^{\prime}}^{A_{2}}$ connected to a mixed monomial in $C_{d^{\prime}}$, we perform the same process obtaining $T_{2}, Y^{b_{2}} \in$ $C_{d^{\prime \prime}}$ with $T_{2}$ as a mixed monomial and $d^{\prime \prime} \prec_{S} d^{\prime}$. This process can be repeated if there existed a pure monomial and a mixed monomial in the same connected component. By degree reasons this cannot be performed indefinitely and an element $d^{(i)} \in \operatorname{Betti}(S)$ verifying that $\nabla_{d^{(i)}}$ is not connected having a connected component with only mixed monomials is found. This contradicts Lemma 3.

After examining the structure of the simplicial complexes associated with glued semigroups, we enunciate a combinatorial characterization by means of the nonconnected simplicial complexes $\nabla_{m}$.

Theorem 6. The semigroup $S$ is the gluing of $S_{1}$ and $S_{2}$ if and only if the following conditions are fulfilled.

(1) For all $d^{\prime} \in \operatorname{Betti}(S)$, any connected component of $\nabla_{d^{\prime}}$ has at least a pure monomial.

(2) There exists a unique $d \in \operatorname{Betti}(S)$ such that $C_{d}^{A_{1}} \neq$ $\emptyset \neq C_{d}^{A_{2}}$ and the elements in $C_{d}$ are pure monomials.

(3) For all $d^{\prime} \in \operatorname{Betti}(S) \backslash\{d\}$ with $C_{d^{\prime}}^{A_{1}} \neq \emptyset \neq C_{d^{\prime}}^{A_{2}}, d^{\prime} \in d \mathbb{N}$.

Besides, the above $d \in \operatorname{Betti}(S)$ is the glued degree.

Proof. If $S$ is the gluing of $S_{1}$ and $S_{2}$, the result is obtained from Lemmas 3, 4, and 5 .

Conversely, by hypotheses 1 and 3, given that $d^{\prime} \epsilon$ $\operatorname{Betti}(S) \backslash\{d\}$, the set $\mathscr{M}\left(I_{S_{1}}\right)_{d^{\prime}}$ is constructed from $C_{d^{\prime}}^{A_{1}}$ and $\mathscr{M}\left(I_{S_{2}}\right)_{d^{\prime}}$ from $C_{d^{\prime}}^{A_{2}}$ as in Construction 1. Analogously, if $d \in \operatorname{Betti}(S)$, the set $\mathscr{M}\left(I_{S}\right)_{d}$ is obtained from the union of $\mathscr{M}\left(I_{S_{1}}\right)_{d}, \mathscr{M}\left(I_{S_{2}}\right)_{d}$ and the binomial $X^{\gamma_{X}}-Y^{\gamma_{Y}}$ with $X^{\gamma_{X}} \in C_{d}^{A_{1}}$ and $Y^{\gamma_{Y}} \in C_{d}^{A_{2}}$. Finally

$$
\coprod_{m \in \operatorname{Betti}(S)}\left(\mathscr{M}\left(I_{S_{1}}\right)_{m} \sqcup \mathscr{M}\left(I_{S_{2}}\right)_{m}\right) \sqcup\left\{X^{\gamma_{X}}-Y^{\gamma_{Y}}\right\}
$$

is a generating set of $I_{S}$ and $S$ is the gluing of $S_{1}$ and $S_{2}$.

From Theorem 6 we obtain an equivalent property to Theorem 12 in [11] by using the language of monomials and binomials.

Corollary 7. Let $S$ be the gluing of $S_{1}$ and $S_{2}$ and $X^{\gamma_{X}}-Y^{\gamma_{Y}} \in I_{S}$ a glued binomial with $S$-degree $d$. The ideal $I_{S}$ is minimally generated by its indispensable binomials if and only if the following conditions are fulfilled.

(i) The ideals $I_{S_{1}}$ and $I_{S_{2}}$ are minimally generated by their indispensable binomials.

(ii) The element $X^{\gamma_{X}}-Y^{\gamma_{Y}}$ is an indispensable binomial of $I_{S}$.

(iii) For all $d^{\prime} \in \operatorname{Betti}(S)$, the elements of $C_{d^{\prime}}$ are pure monomials.

Proof. Suppose that $I_{S}$ is generated by its indispensable binomials. By [10, Corollary 6], for all $m \in \operatorname{Betti}(S)$ the simplicial complex $\nabla_{m}$ has only two vertices. By Construction $1 \nabla_{d}=\left\{\left\{X^{\gamma_{X}}\right\},\left\{Y^{\gamma_{Y}}\right\}\right\}$ and by Theorem 6 for all $d^{\prime} \in \operatorname{Betti}(S) \backslash$ $\{d\}$ the simplicial $\nabla_{d^{\prime}}$ is equal to $\nabla_{d^{\prime}}^{A_{1}}$ or $\nabla_{d^{\prime}}^{A_{2}}$. In any case, $X^{\gamma_{X}}-Y^{\gamma_{Y}} \in I_{S}$ is an indispensable binomial, and $I_{S_{1}}, I_{S_{2}}$ are generated by their indispensable binomials.

Conversely, suppose that $I_{S}$ is not generated by its indispensable binomials. Then, there exists $d^{\prime} \in \operatorname{Betti}(S) \backslash\{d\}$ such that $\nabla_{d^{\prime}}$ has more than two vertices in at least two different connected components. By hypothesis, there are not mixed monomials in $\nabla_{d^{\prime}}$ and thus

(i) if $\nabla_{d^{\prime}}$ is equal to $\nabla_{d^{\prime}}^{A_{1}}\left(\right.$ or $\left.\nabla_{d^{\prime}}^{A_{2}}\right)$, then $I_{S_{1}}\left(\right.$ or $\left.I_{S_{2}}\right)$ is not generated by its indispensable binomials;

(ii) otherwise, $C_{d^{\prime}}^{A_{1}} \neq \emptyset \neq C_{d^{\prime}}^{A_{2}}$ and by Lemma $4, d^{\prime}=j d$ with $j \in \mathbb{N}$, therefore $X^{(j-1) \gamma_{X}} Y^{\gamma_{Y}} \in C_{d^{\prime}}$ which contradicts the hypothesis.

We conclude that $I_{S}$ is generated by its indispensable binomials. results.

The following example taken from [5] illustrates the above Example 8. Let $S \subset \mathbb{N}^{2}$ be the semigroup generated by the set

$$
\{(13,0),(5,8),(2,11),(0,13),(4,4),(6,6),(7,7),(9,9)\} \text {. }
$$

In this case, $\operatorname{Betti}(S)$ is

$$
\begin{aligned}
& \{(15,15),(14,14),(12,12),(18,18), \\
& (10,55),(15,24),(13,52),(13,13)\} .
\end{aligned}
$$

Using the appropriated notation for the indeterminates in the polynomial ring $\mathbb{K}\left[x_{1}, \ldots, x_{4}, y_{1}, \ldots, y_{4}\right]\left(x_{1}, x_{2}, x_{3}\right.$, and $x_{4}$ for the first four generators of $S$ and $y_{1}, y_{2}, y_{3}, y_{4}$ for the others), the simplicial complexes associated with the elements in $\operatorname{Betti}(S)$ are those that appear in Figure 1. From Figure 1 and by using Theorem 6 , the semigroup $S$ is the gluing of $\langle(13,0),(5,8),(2,11),(0,13)\rangle$ and $\langle(4,4),(6,6),(7,7),(9,9)\rangle$ and the glued degree is $(13,13)$. From Corollary 7 , the ideal $I_{S}$ is not generated by its indispensable binomials $\left(I_{S}\right.$ has only four indispensable binomials). 


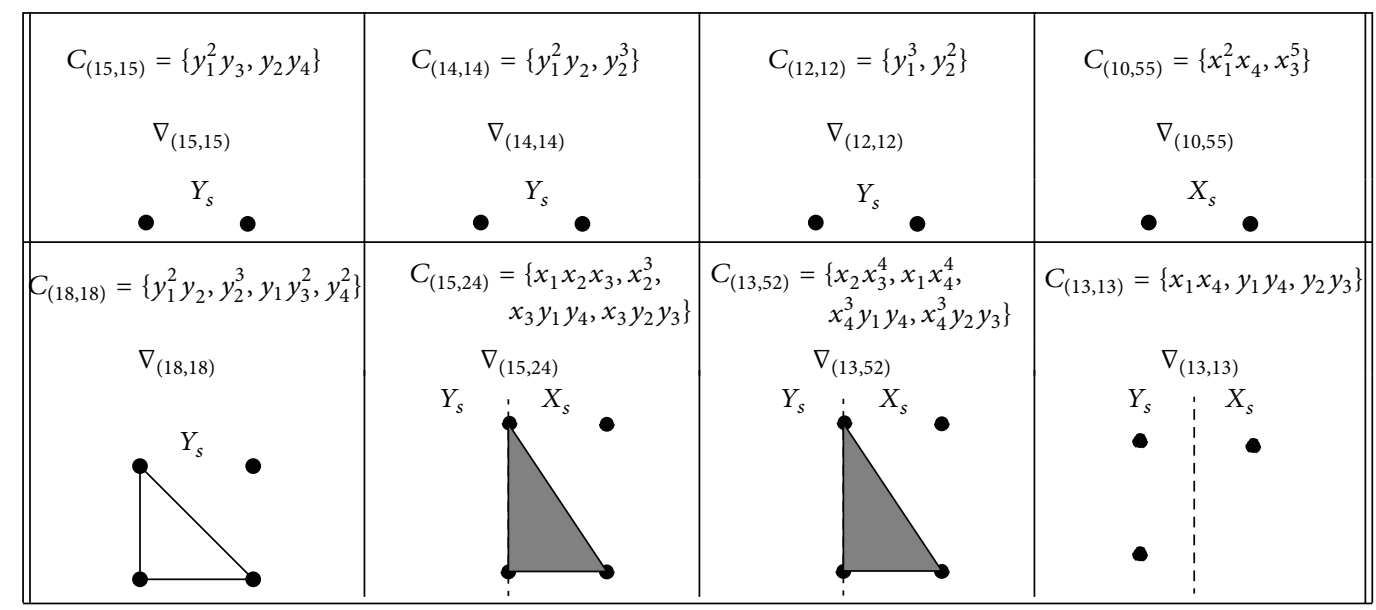

Figure 1: Nonconnected simplicial complexes associated with $\operatorname{Betti}(S)$.

\section{Generating Glued Semigroups}

In this section, an algorithm to obtain examples of glued semigroups is given. Consider $A_{1}=\left\{a_{1}, \ldots, a_{r}\right\}$ and $A_{2}=$ $\left\{b_{1}, \ldots, b_{t}\right\}$ as two minimal generator sets of the semigroups $T_{1}$ and $T_{2}$ and let $L_{j}=\left\{\rho_{j i}\right\}_{i}$ be a basis of $\operatorname{ker} T_{j}$ with $j=1,2$. Assume that $I_{T_{1}}$ and $I_{T_{2}}$ are nontrivial proper ideals of their corresponding polynomial rings. Consider $\gamma_{X}$ and $\gamma_{Y}$ be two nonzero elements in $\mathbb{N}^{r}$ and $\mathbb{N}^{t}$, respectively, (note that $\gamma_{X} \notin$ $\operatorname{ker} T_{1}$ and $\gamma_{Y} \notin \operatorname{ker} T_{2}$ because these semigroups are reduced) and the integer matrix

$$
A=\left(\begin{array}{c|c}
L_{1} & 0 \\
\hline 0 & L_{2} \\
\hline \gamma_{X} & -\gamma_{Y}
\end{array}\right) .
$$

Let $S$ be a semigroup such that $\operatorname{ker} S$ is the lattice generated by the rows of matrix $A$. This semigroup can be computed by using the Smith Normal Form (see [1, Chapter 3]). Denote by $B_{1}, B_{2}$ two sets of cardinality $r$ and $t$, respectively, satisfying $S=\left\langle B_{1}, B_{2}\right\rangle$ and $\operatorname{ker}\left(\left\langle B_{1}, B_{2}\right\rangle\right)$ is generated by the rows of $A$.

The following proposition shows that the semigroup $S$ satisfies one of the necessary conditions to be a glued semigroup.

Proposition 9. The semigroup $S$ verifies $G\left(\left\langle B_{1}\right\rangle\right) \cap G\left(\left\langle B_{2}\right\rangle\right)=$ $\left(B_{1} \gamma_{X}\right) \mathbb{Z}=\left(B_{2} \gamma_{Y}\right) \mathbb{Z}$ with $d=B_{1} \gamma_{X} \in\left\langle B_{1}\right\rangle \cap\left\langle B_{2}\right\rangle$.

Proof. Use that ker $S$ has a basis as (4) and proceed as in the proof of the necessary condition of Proposition 2.

Because $B_{1} \cup B_{2}$ may not be a minimal generating set, this condition does not assure that $S$ is a glued semigroup. For instance, taking the numerical semigroups $T_{1}=\langle 3,5\rangle$, $T_{2}=\langle 2,7\rangle$, and $\left(\gamma_{X}, \gamma_{Y}\right)=(1,0,2,0)$, the matrix obtained from formula (17) is

$$
\left(\begin{array}{cc|cc}
5 & -3 & 0 & 0 \\
\hline 0 & 0 & 7 & -2 \\
\hline 1 & 0 & -2 & 0
\end{array}\right),
$$

and $B_{1} \cup B_{2}=\{12,20,6,21\}$ is not a minimal generating set. The following result solves this issue.
Corollary 10. The semigroup $S$ is a glued semigroup if

$$
\sum_{i=1}^{r} \gamma_{X i}>1, \quad \sum_{i=1}^{t} \gamma_{Y i}>1 .
$$

Proof. Suppose that the set of generators $B_{1} \cup B_{2}$ of $S$ is nonminimal and thus one of its elements is a natural combination of the others. Assume that this element is the first of $B_{1} \cup B_{2}$ and then there exist $\lambda_{2}, \ldots, \lambda_{r+t} \in \mathbb{N}$ such that $B_{1}\left(1,-\lambda_{2}, \ldots,-\lambda_{r}\right)=B_{2}\left(\lambda_{r+1}, \ldots, \lambda_{r+t}\right) \in G\left(\left\langle B_{1}\right\rangle\right) \cap$ $G\left(\left\langle B_{2}\right\rangle\right)$. By Proposition 9 , there exists $\lambda \in \mathbb{Z}$ satisfying $B_{1}\left(1,-\lambda_{2}, \ldots,-\lambda_{r}\right)=B_{2}\left(\lambda_{r+1}, \ldots, \lambda_{r+t}\right)=B_{1}\left(\lambda \gamma_{X}\right)$. Since $B_{2}\left(\lambda_{r+1}, \ldots, \lambda_{r+t}\right) \in S, \lambda \geq 0$ and thus

$$
\begin{aligned}
v & =(1-\lambda \gamma_{X 1}, \underbrace{-\lambda_{2}-\lambda \gamma_{X 2}, \ldots,-\lambda_{r}-\lambda \gamma_{X r}}_{\leq 0}) \\
& \in \operatorname{ker}\left(\left\langle B_{1}\right\rangle\right)=\operatorname{ker} T_{1},
\end{aligned}
$$

with the following cases.

(i) If $\lambda \gamma_{X 1}=0$, then $T_{1}$ is not minimally generated which it is not possible by hypothesis.

(ii) If $\lambda \gamma_{X 1}>1$, then $0>v \in \operatorname{ker} T_{1}$, but this is not possible because $T_{1}$ is a reduced semigroup.

(iii) If $\lambda \gamma_{X 1}=1$, then $\lambda=\gamma_{X 1}=1$ and

$$
\nu=(0, \underbrace{-\lambda_{2}-\gamma_{X 2}, \ldots,-\lambda_{r}-\gamma_{X r}}_{\leq 0}) \in \operatorname{ker} T_{1} .
$$

If $\lambda_{i}+\gamma_{X i} \neq 0$ for some $i=2, \ldots, r$, then $T_{1}$ is not a reduced semigroup. This implies that $\lambda_{i}=\gamma_{X i}=0$ for all $i=2, \ldots, r$.

We have just proved that $\gamma_{X}=(1,0, \ldots, 0)$. In the general case, if $S$ is not minimally generated it is because either $\gamma_{X}$ or $\gamma_{Y}$ are elements in the canonical bases of $\mathbb{N}^{r}$ or $\mathbb{N}^{t}$, respectively. To avoid this situation, it is sufficient to take $\gamma_{X}$ and $\gamma_{Y}$ satisfying $\sum_{i=1}^{r} \gamma_{X i}>1$ and $\sum_{i=1}^{t} \gamma_{Y i}>1$. 
From the above result we obtain a characterization of glued semigroups: $S$ is a glued semigroup if and only if ker $S$ has a basis as (4) satisfies Condition (19).

Example 11. Let $T_{1}=\langle(-7,2),(11,1),(5,0),(0,1)\rangle \subset \mathbb{Z}^{2}$ and $T_{2}=\langle 3,5,7\rangle \subset \mathbb{N}$ be two reduced affine semigroups. We compute their associated lattices

$$
\begin{aligned}
& \operatorname{ker} T_{1}=\langle(1,2,-3,-4),(2,-1,5,-3)\rangle, \\
& \operatorname{ker} T_{2}=\langle(-4,1,1),(-7,0,3)\rangle .
\end{aligned}
$$

If we take $\gamma_{X}=(2,0,2,0)$ and $\gamma_{Y}=(1,2,1)$, the matrix $A$ is

$$
\left(\begin{array}{ccccccc}
1 & 2 & -3 & -4 & 0 & 0 & 0 \\
2 & -1 & 5 & -3 & 0 & 0 & 0 \\
0 & 0 & 0 & 0 & -4 & 1 & 1 \\
0 & 0 & 0 & 0 & -7 & 0 & 3 \\
2 & 0 & 2 & 0 & -1 & -2 & -1
\end{array}\right)
$$

and the semigroup $S \subset \mathbb{Z}_{4} \times \mathbb{Z}^{2}$ is generated by

$$
\begin{aligned}
& \{\underbrace{(1,-5,35),(3,12,-55),(1,5,-25),(0,1,0)}_{B_{1}}, \\
& \underbrace{(2,0,3),(2,0,5),(2,0,7)}_{B_{2}}\} .
\end{aligned}
$$

The semigroup $S$ is the gluing of the semigroups $\left\langle B_{1}\right\rangle$ and $\left\langle B_{2}\right\rangle$ and $\operatorname{ker} S$ is generated by the rows of the above matrix. The ideal $I_{S} \subset \mathbb{C}\left[x_{1}, \ldots, x_{4}, y_{1}, \ldots, y_{3}\right]$ is generated (see [12] to compute $I_{S}$ when $S$ has torsion) by

$$
\begin{gathered}
\left\{x_{1} x_{3}^{8} x_{4}-x_{2}^{3}, x_{1} x_{2}^{2}-x_{3}^{3} x_{4}^{4}, x_{1}^{2} x_{3}^{5}-x_{2} x_{4}^{3}, x_{1}^{3} x_{2} x_{3}^{2}-x_{7}^{7}\right. \\
y_{1} y_{3}-y_{2}^{2}, y_{1}^{3} y_{2}-y_{3}^{2}, y_{1}^{4}-y_{2} y_{3}, \underbrace{x_{1}^{2} x_{3}^{2}-y_{1}^{5} y_{2}}_{\text {glued binomial }}\}
\end{gathered}
$$

then $S$ is really a glued semigroup.

4.1. Generating Affine Glued Semigroups. From Example 11 it be can deduced that the semigroup $S$ is not necessarily torsion-free. In general, a semigroup $T$ is affine (or equivalently it is torsion-free) if and only if the invariant factors (the invariant factors of a matrix are the diagonal elements of its Smith Normal Form (see [13, Chapter 2] and [1, Chapter 2])) of the matrix whose rows are a basis of $\operatorname{ker} T$ are equal to one. Assume that zero-columns of the Smith Normal Form of a matrix are located on its right side. We now show conditions for $S$ being torsion-free.
Take $L_{1}$ and $L_{2}$ as the matrices whose rows form a basis of $\operatorname{ker} T_{1}$ and $\operatorname{ker} T_{2}$, respectively, and let $P_{1}, P_{2}, Q_{1}$, and $Q_{2}$ be some matrices with determinant \pm 1 (i.e., unimodular matrices) such that $D_{1}=P_{1} L_{1} Q_{1}$ and $D_{2}=P_{2} L_{2} Q_{2}$ are the Smith Normal Form of $L_{1}$ and $L_{2}$, respectively. If $T_{1}$ and $T_{2}$ are two affine semigroups, the invariant factors of $L_{1}$ and $L_{2}$ are equal to 1 . Then

$$
\left(\begin{array}{c|c}
D_{1} & 0 \\
\hline 0 & D_{2} \\
\hline \gamma_{X}^{\prime} & \gamma_{Y}^{\prime}
\end{array}\right)=\left(\begin{array}{c|c|c}
P_{1} & 0 & 0 \\
\hline 0 & P_{2} & 0 \\
\hline 0 & 0 & 1
\end{array}\right) \underbrace{\left(\begin{array}{c|c}
L_{1} & 0 \\
\hline 0 & L_{2} \\
\hline \gamma_{X} & -\gamma_{Y}
\end{array}\right)}_{=: A}\left(\begin{array}{c|c}
Q_{1} & 0 \\
\hline 0 & Q_{2}
\end{array}\right),
$$

where $\gamma_{X}^{\prime}=\gamma_{X} Q_{1}$ and $\gamma_{Y}^{\prime}=-\gamma_{Y} Q_{2}$. Let $s_{1}$ and $s_{2}$ be the numbers of zero-columns of $D_{1}$ and $D_{2}\left(s_{1}, s_{2}>0\right.$ because $T_{1}$ and $T_{2}$ are reduced, see [1, Theorem 3.14]).

Lemma 12. The semigroup $S$ is an affine semigroup if and only if

$$
\operatorname{gcd}\left(\left\{\gamma_{X i}^{\prime}\right\}_{i=r-s_{1}}^{r} \cup\left\{\gamma_{Y i}^{\prime}\right\}_{i=t-s_{2}}^{t}\right)=1
$$

Proof. With the conditions fulfilled by $T_{1}, T_{2}$, and $\left(\gamma_{X}, \gamma_{Y}\right)$, the necessary and sufficient condition for the invariant factors of $A$ to be all equal to one is $\operatorname{gcd}\left(\left\{\gamma_{X i}^{\prime}\right\}_{i=r-s_{1}}^{r} \cup\left\{\gamma_{Y i}^{\prime}\right\}_{i=t-s_{2}}^{t}\right)=1$.

The following corollary gives the explicit conditions that $\gamma_{X}$ and $\gamma_{Y}$ must satisfy to construct an affine semigroup.

Corollary 13. The semigroup $S$ is an affine glued semigroup if and only if

(1) $T_{1}$ and $T_{2}$ are two affine semigroups;

(2) $\left(\gamma_{X}, \gamma_{Y}\right) \in \mathbb{N}^{r+t}$;

(3) $\sum_{i=1}^{r} \gamma_{X i}, \sum_{i=1}^{t} \gamma_{Y i}>1$;

(4) there exist $f_{r-s_{1}}, \ldots, f_{r}, g_{t-s_{2}}, \ldots, g_{t} \in \mathbb{Z}$ such that

$$
\begin{aligned}
& \left(f_{r-s_{1}}, \ldots, f_{r}\right) \cdot\left(\gamma_{X\left(r-s_{1}\right)}^{\prime}, \ldots, \gamma_{X r}^{\prime}\right) \\
& \quad+\left(g_{t-s_{2}}, \ldots, g_{t}\right) \cdot\left(\gamma_{Y\left(t-s_{2}\right)}^{\prime}, \ldots, \gamma_{Y t}^{\prime}\right)=1 .
\end{aligned}
$$

Proof. It is trivial by the given construction, Corollary 10 and Lemma 12.

Therefore, to obtain an affine glued semigroup it is enough to take two affine semigroups and any solution $\left(\gamma_{X}\right.$, $\gamma_{Y}$ ) of the equations of the above corollary.

Example 14. Let $T_{1}$ and $T_{2}$ be the semigroups of Example 11. We compute two elements $\gamma_{X}=\left(a_{1}, a_{2}, a_{3}, a_{4}\right)$ and $\gamma_{Y}=$ $\left(b_{1}, b_{2}, b_{3}\right)$ in order to obtain an affine semigroup. First of all, we perform a decomposition of the matrix as (26) by computing the integer Smith Normal Form of $L_{1}$ and $L_{2}$ : 


$$
\begin{aligned}
& \left(\begin{array}{cccc|ccc}
1 & 0 & 0 & 0 & 0 & 0 & 0 \\
0 & 1 & 0 & 0 & 0 & 0 & 0 \\
\hline 0 & 0 & 0 & 0 & 1 & 0 & 0 \\
0 & 0 & 0 & 0 & 0 & 1 & 0 \\
\hline a_{1} & a_{1}-2 a_{2}-a_{3} & -7 a_{1}+11 a_{2}+5 a_{3} & 2 a_{1}+a_{2}+a_{4} & -b_{1} & b_{1}+2 b_{2}+3 b_{3} & -3 b_{1}-5 b_{2}-7 b_{3}
\end{array}\right) \\
& =\left(\begin{array}{cc|cc|c}
1 & 0 & 0 & 0 & 0 \\
2 & -1 & 0 & 0 & 0 \\
\hline 0 & 0 & -2 & 1 & 0 \\
0 & 0 & 7 & -4 & 0 \\
\hline 0 & 0 & 0 & 0 & 1
\end{array}\right)\left(\begin{array}{cccc|ccc}
1 & 2 & -3 & -4 & 0 & 0 & 0 \\
2 & -1 & 5 & -3 & 0 & 0 & 0 \\
\hline 0 & 0 & 0 & 0 & -4 & 1 & 1 \\
0 & 0 & 0 & 0 & -7 & 0 & 3 \\
\hline a_{1} & a_{2} & a_{3} & a_{4} & -b_{1} & -b_{2} & -b_{3}
\end{array}\right)\left(\begin{array}{cccc|ccc}
1 & 1 & -7 & 2 & 0 & 0 & 0 \\
0 & -2 & 11 & 1 & 0 & 0 & 0 \\
0 & -1 & 5 & 0 & 0 & 0 & 0 \\
0 & 0 & 0 & 1 & 0 & 0 & 0 \\
\hline 0 & 0 & 0 & 0 & 1 & -1 & 3 \\
0 & 0 & 0 & 0 & 0 & -2 & 5 \\
0 & 0 & 0 & 0 & 0 & -3 & 7
\end{array}\right) .
\end{aligned}
$$

Second, by Corollary 13, we must find a solution to the system:

$$
\begin{gathered}
a_{1}+a_{2}+a_{3}+a_{4}>1, \\
b_{1}+b_{2}+b_{3}>1, \\
f_{1}, f_{2}, g_{1} \in \mathbb{Z}, \\
f_{1}\left(-7 a_{1}+11 a_{2}+5 a_{3}\right)+f_{2}\left(2 a_{1}+a_{2}+a_{4}\right) \\
+g_{1}\left(-3 b_{1}-5 b_{2}-7 b_{3}\right)=1,
\end{gathered}
$$

with $a_{1}, a_{2}, a_{3}, a_{4}, b_{1}, b_{2}, b_{3} \in \mathbb{N}$. Such solution is computed (in less than a second) using FindInstance of Wolfram Mathematica (see [14]):

$$
\begin{aligned}
& \text { FindInstance }\left[\left(-7 a_{1}+11 a_{2}+5 a_{3}\right) * f_{1}\right. \\
& +\left(2 a_{1}+a_{2}+a_{4}\right) * f_{2} \\
& +\left(-3 b_{1}-5 b_{2}-7 b_{3}\right) * g_{1}==1 \\
& \& \& a 1+a 2+a 3+a 4>1 \\
& \& \& b_{1}+b_{2}+b_{3}>1 \& \& a_{1} \geq 0 \& \& a_{2} \geq 0 \\
& \& \& a_{3} \geq 0 \& \& a_{4} \geq 0 \& \& b_{1} \geq 0 \\
& \& \& b_{2} \geq 0 \& \& b_{3} \geq 0 \text {, } \\
& \left\{a_{1}, a_{2}, a_{3}, a_{4}, b_{1}, b_{2}, b_{3}, f_{1}, f_{2}, g_{1}\right\} \text {, } \\
& \text { Integers] }
\end{aligned}
$$$$
\downarrow
$$$$
\begin{aligned}
& \left\{\left\{a_{1} \longrightarrow 0, a_{2} \longrightarrow 0, a_{3} \longrightarrow 3, a_{4} \longrightarrow 0, b_{1} \longrightarrow 1\right.\right. \\
& \left.\left.\quad b_{2} \longrightarrow 1, b_{3} \longrightarrow 0, f_{1} \longrightarrow 1, f_{2} \longrightarrow 0, g_{1} \longrightarrow 0\right\}\right\}
\end{aligned}
$$

We now take $\gamma_{X}=(0,0,3,0)$ and $\gamma_{Y}=(1,1,0)$, and construct the matrix

$$
A=\left(\begin{array}{ccccccc}
1 & 2 & -3 & -4 & 0 & 0 & 0 \\
2 & -1 & 5 & -3 & 0 & 0 & 0 \\
0 & 0 & 0 & 0 & -4 & 1 & 1 \\
0 & 0 & 0 & 0 & -7 & 0 & 3 \\
0 & 0 & 3 & 0 & -1 & -1 & 0
\end{array}\right)
$$

We have the affine semigroup $S \subset \mathbb{Z}^{2}$ which is minimally generated by

$$
\{\underbrace{(2,-56),(1,88),(0,40),(1,0)}_{B_{1}}, \underbrace{(0,45),(0,75),(0,105)}_{B_{2}}\}
$$

satisfying that $\operatorname{ker} S$ is generated by the rows of $A$ and it is the result of gluing the semigroups $\left\langle B_{1}\right\rangle$ and $\left\langle B_{2}\right\rangle$. The ideal $I_{S}$ is generated by

$$
\begin{aligned}
& \left\{x_{1} x_{3}^{8} x_{4}-x_{2}^{3}, x_{1} x_{2}^{2}-x_{3}^{3} x_{4}^{4}, x_{1}^{2} x_{3}^{5}-x_{2} x_{4}^{3}, x_{1}^{3} x_{2} x_{3}^{2}-x_{4}^{7},\right. \\
& y_{1} y_{3}-y_{2}^{2}, y_{1}^{3} y_{2}-y_{3}^{2}, y_{1}^{4}-y_{2} y_{3}, \underbrace{x_{3}^{3}-y_{1} y_{2}}_{\text {glued binomial }}\}
\end{aligned}
$$

therefore, $S$ is a glued semigroup.

All glued semigroups have been computed by using our program Ecuaciones which is available in [15] (this program requires Wolfram Mathematica 7 or above to run).

\section{Conflict of Interests}

The authors declare that there is no conflict of interests regarding the publication of this paper.

\section{Acknowledgments}

J. I. García-García was partially supported by MTM201015595 and Junta de Andalucía group FQM-366. 
M. A. Moreno-Frías was partially supported by MTM200806201-C02-02 and Junta de Andalucía group FQM-298. A. Vigneron-Tenorio was partially supported by Grant MTM2007-64704 (with the help of FEDER Program), MTM2012-36917-C03-01, and Junta de Andalucía group FQM-366.

\section{References}

[1] J. C. Rosales and P. A. García-Sánchez, Finitely Generated Commutative Monoids, Nova Science Publishers, New York, NY, USA, 1999.

[2] J. Herzog, "Generators and relations of abelian semigroups and semigroup rings," Manuscripta Mathematica, vol. 3, no. 2, pp. 175-193, 1970.

[3] B. Sturmfels, Gröbner Bases and Convex Polytopes, vol. 8 of University Lecture, American Mathematical Society, Providence, RI, USA, 1996.

[4] J. C. Rosales, "On presentations of subsemigroups of $\mathbb{N}^{n}$," Semigroup Forum, vol. 55, no. 2, pp. 152-159, 1997.

[5] A. Thoma, "Construction of set theoretic complete intersections via semigroup gluing," Beitrage zur Algebra und Geometrie, vol. 41, no. 1, pp. 195-198, 2000.

[6] E. Briales, A. Campillo, C. Marijuán, and P. Pisón, "Minimal systems of generators for ideals of semigroups," Journal of Pure and Applied Algebra, vol. 124, no. 1-3, pp. 7-30, 1998.

[7] S. Eliahou, Courbes monomiales et algébre de Rees symbolique [Ph.D. thesis], Université of Genève, Genève, Switzerland, 1983.

[8] I. Ojeda and A. Vigneron-Tenorio, "Simplicial complexes and minimal free resolution of monomial algebras," Journal of Pure and Applied Algebra, vol. 214, no. 6, pp. 850-861, 2010.

[9] H. Ohsugi and T. Hibi, "Toric ideals arising from contingency tables," in Commutative Algebra and Combinatorics, vol. 4 of Ramanujan Mathematical Society Lecture Notes Series, pp. 91115, Ramanujan Mathematical Society, Mysore, India, 2007.

[10] I. Ojeda and A. Vigneron-Tenorio, "Indispensable binomials in semigroup ideals," Proceedings of the American Mathematical Society, vol. 138, no. 12, pp. 4205-4216, 2010.

[11] P. A. García-Sánchez and I. Ojeda, "Uniquely presented finitely generated commutative monoids," Pacific Journal of Mathematics, vol. 248, no. 1, pp. 91-105, 2010.

[12] A. Vigneron-Tenorio, "Semigroup ideals and linear diophantine equations," Linear Algebra and Its Applications, vol. 295, no. 1-3, pp. 133-144, 1999.

[13] H. Cohen, A Course in Computational Algebraic Number Theory, vol. 138 of Graduate Texts in Mathematics, Springer, New York, NY, USA, 1996.

[14] Wolfram Mathematica, http://www.wolfram.com/mathematica/.

[15] Ecuaciones, http://www.uca.es/dpto/C101/pags-personales/alberto.vigneron/lecuaciones.rar. 


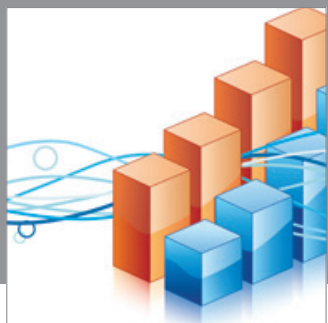

Advances in

Operations Research

mansans

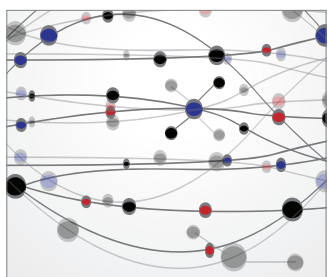

The Scientific World Journal
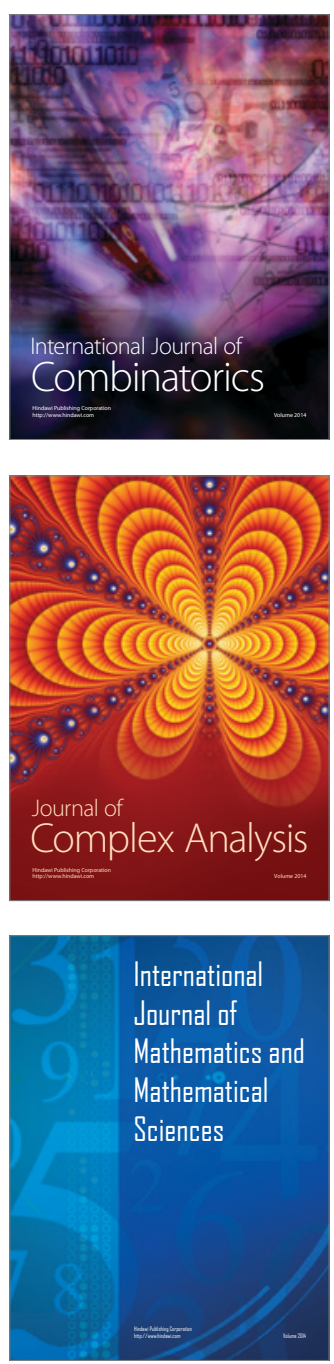
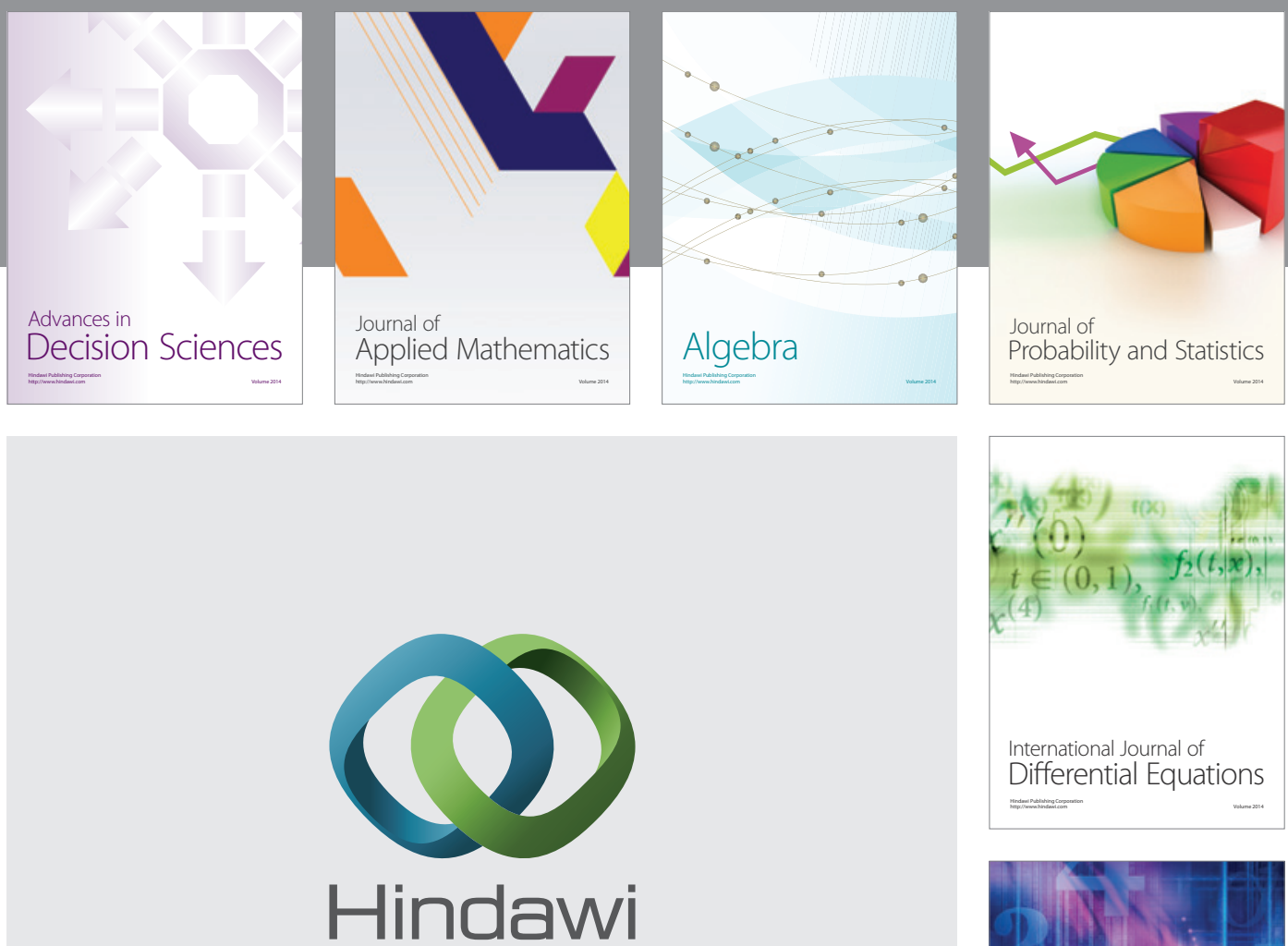

Submit your manuscripts at http://www.hindawi.com
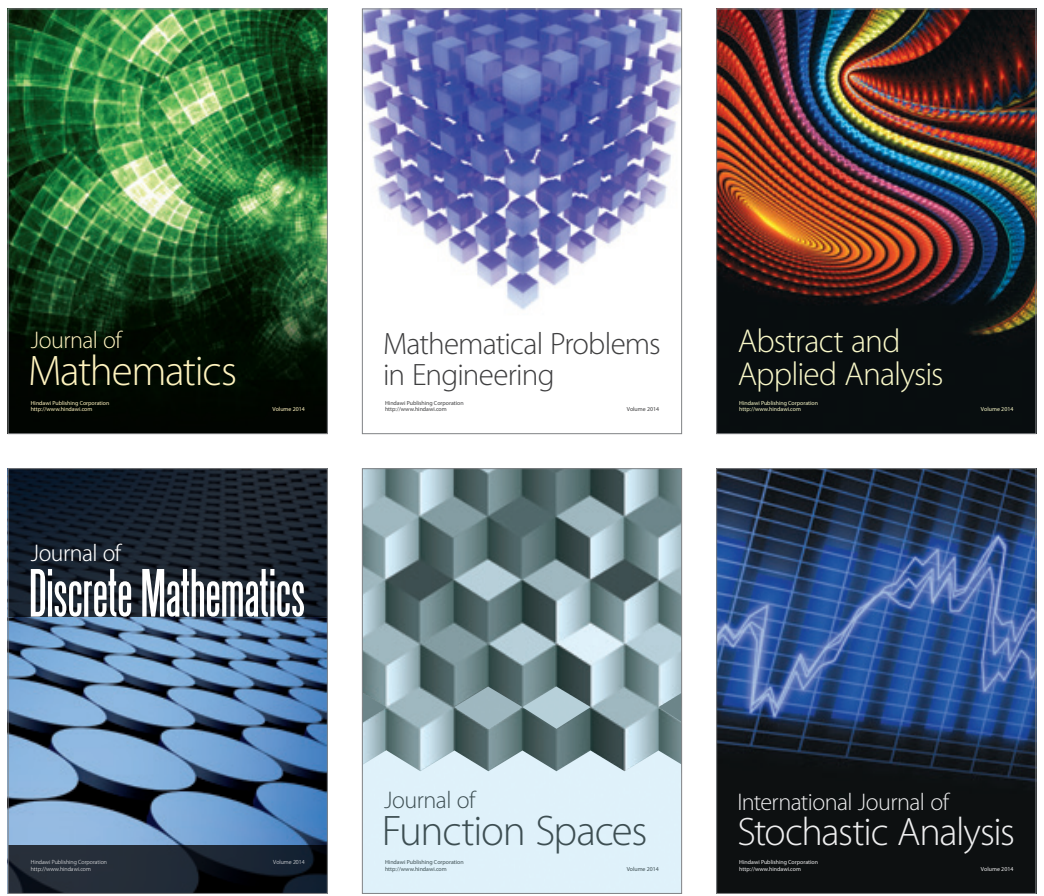

Journal of

Function Spaces

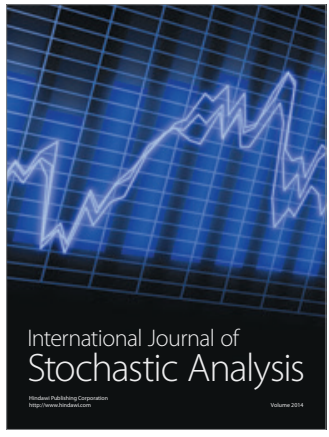

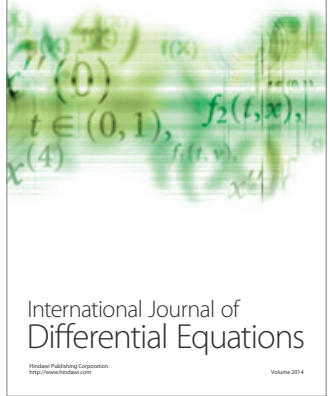
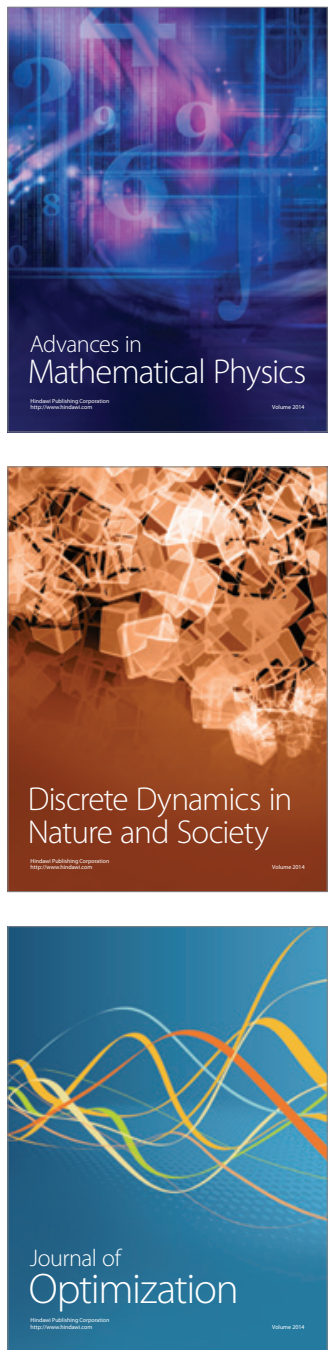